\title{
WEAK LIMITS OF MEASURES AND THE STANDARD PART MAP
}

\author{
PETER A. LOEB ${ }^{1}$
}

\begin{abstract}
A construction is given, using the standard part map st, of the weak* standard part of an internal Baire measure in the nonstandard extension of a compact Hausdorff space. It is shown that the inverse image with respect to st of a Borel set is universally measurable with respect to completions of the $\sigma$-algebra generated by internal Baire sets. Applications and extensions of these results to noncompact spaces are given.
\end{abstract}

In this note, we give a construction of the weak* standard part for a finite-valued, internal Baire measure in the nonstandard extension ${ }^{*} X$ (see [15] or [17]) of a compact Hausdorff space $X$. The construction uses the measurability of the standard part map st: ${ }^{*} X \rightarrow X$; i.e., for each Baire set $B \subset X, \mathrm{st}^{-1}(B)$ is in the external $\sigma$-algebra $\sigma(* \mathscr{B}(X))$ generated by the internal Baire sets in ${ }^{*} X$. We shall also show that for each Borel set $A \subset X$, $\mathrm{st}^{-1}(A)$ is "universally" measurable, i.e., $\mathrm{st}^{-1}(A)$ is in the completion of $\sigma\left({ }^{*} \mathscr{B}(X)\right)$ with respect to any internal, finite-valued Baire measure on ${ }^{*} X$. These results have extensions to noncompact spaces and are related, as we shall show, to results of Anderson and Rashid in [3].

Let $(X, \mathcal{T})$ be a compact Hausdorff space, $C(X)$ the continuous real-valued functions on $X, \mathscr{B}(X)$ the class of Baire subsets of $X$, and $M(X)$ the space of signed Baire measures $\mu$ on $X$ with finite norm

$$
\|\mu\|=|\mu|(X)=\mu^{+}(X)+\mu^{-}(X) .
$$

Fix a $\kappa$-saturated enlargement [11] of a structure containing $X$ and the real numbers $R$, with $\kappa>\max \left(\kappa_{0}\right.$, card $\left.\mathcal{T}\right)$. Let $\sigma(* \mathscr{B}(X))$ be the smallest ordinary $\sigma$-algebra of subsets of ${ }^{*} X$ containing the class ${ }^{*} \mathscr{B}(X)$ of internal Baire sets in ${ }^{*} X$. We let ${ }^{*} M_{f}(X)$ denote the set of $\nu \in{ }^{*} M(X)$ with ${ }^{0}(\|\nu\|)<$ $+\infty$. Given $\nu \in{ }^{*} M_{f}(X)$, let $\nu_{0}(A)={ }^{0}(\nu(A))$ for each $A \in \mathscr{B}^{*}(X)$. By Theorem 1 of $[8], \nu_{0}$ has a unique, countably additive, real-valued extension $\nu_{0}$ defined on $\sigma(* \mathscr{B}(X))$. We shall use $\nu_{0}$ to construct the weak* standard part of $\nu$, i.e., the unique $\mu \in M(X)$ such that for each $f \in C(X)$,

Received by the editors November 3, 1978.

AMS (MOS) subject classifications (1970). Primary 02H25, 26A98, 28A10; Secondary 60B05, $60 \mathrm{~B} 10$.

Key words and phrases. Nonstandard analysis, weak* standard part, weak* cluster point, Kolmogorov extension theorem, Riesz-Herglotz theorem, tight measure.

${ }^{1}$ This research was supported by the U. S. National Science Foundation. The author is indebted to Ward Henson for several helpful conversations. 


$$
\int_{*^{*}}{ }^{*} f d \nu=\int_{X} f d \mu
$$

In Theorem 4.6 of [9], maximal representing measures for positive harmonic functions were obtained by taking internal representing measures $\nu$ on the space of nonstandard harmonic functions and transforming the corresponding measures $\nu_{0}$ via the (measurable) standard part map into representing measures on the space of standard harmonic functions. That the images were representing measures followed from the fact that integration with respect to point evaluations was preserved, a fact established using only the continuity of point evaluations. Subsequently, Anderson [1] used the standard part map to obtain Lebesque measure on $[0,1]$ and Wiener measure on $C[0,1]$. The author then added to [9] the following construction which suggested Theorem 1 of this paper and shows the need to consider in its formulation arbitrary internal subsets $Z$ of ${ }^{*} X$.

Example 1. Let $D_{r}$ be the open disk $\{z \in \mathbf{C}:|z|<r\}$ with closure $\bar{D}_{r}$. Let $P(z, a)$ denote the Poisson kernel $\left(|z|^{2}-|a|^{2}\right) /|z-a|^{2}$ on $\bar{D}_{1}$ and let $\lambda_{r}$ denote Lebesgue measure divided by $2 \pi r$ on $T_{r}=\bar{D}_{r}-D_{r}$. It is well known ([6] and [14]) that for each harmonic function $h>0$ on $D_{1}$, the measures $h \cdot \lambda_{r}$ converge in the weak * topology as $r \rightarrow 1$ to a unique measure $\nu_{h}$ on $T_{1}$ such that for each $a \in D_{1}$,

$$
h(a)=\int_{T_{1}} P(z, a) d \nu_{h}(z) .
$$

As in [9], we fix $r \in{ }^{*} R$ with $r<1, r \simeq 1$, and we let $\mu$ be the image of $\left(h \cdot \lambda_{r}\right)_{0}$ via the standard part map from $T_{r}$ onto $T_{1}$. By Theorem 6.5 of [9], $\mu=\nu_{h}$. Note that $\nu_{h}$ is the weak* standard part of $h \cdot \lambda_{r}$.

The validity of this construction of $\nu_{h}$ (and its generalization in [10]) can be established in the following general setting: Fix an internal subset $Z$ of ${ }^{*} X$, and let $\mathbb{Q}$ denote the algebra of internal Baire sets in $Z$. That is, $\mathcal{Q}$ is the smallest internal $\sigma$-algebra (here, $\sigma$ refers to ${ }^{*} N$ ) for which the internal bounded and continuous functions are internally measurable. Again, $\sigma(\mathbb{Q})$ is the smallest $\sigma$-algebra (in the ordinary sense) in $Z$ containing $Q$. Let $\nu$ be an internal, nonnegative $\mathcal{Q}$-measure on $Z$ with ${ }^{0}(\nu(Z))<+\infty$, and let $\nu_{0}$ be the corresponding $\sigma$-additive measure on $(Z, \sigma(\mathbb{Q}))$ obtained as in [8]. By Theorem 3.4.2 of [11], the set $K=\operatorname{st}(Z)$ is compact in $X$. Let $\mathscr{B}(K)$ denote the Baire sets in $K$.

THEOREM 1. The standard part map $\mathrm{st}_{Z}: Z \rightarrow K$, where $\mathrm{st}_{Z}(x)={ }^{0} x$ for each $x \in Z$, is measurable with respect to $\sigma(\mathscr{Q})$ and $\mathscr{B}(K)$. Let $\nu_{K}$ be the Baire measure on $K$ obtained by setting $\nu_{K}(B)=\nu_{0}\left(\mathrm{st}_{Z}^{-1}(B)\right)$ for each $B \in \mathscr{B}(K)$. Then for each $f \in C(X)$,

$$
\int_{K} f(x) d \nu_{K}(x)=\int_{Z}{ }^{0}\left({ }^{*} f(z)\right) d \nu_{0}(z) \simeq \int_{Z}{ }^{*} f(z) d \nu(z) .
$$


Proof. The set $\left\{B \subset K\right.$ : $\left.\operatorname{st}_{Z}^{-1}(B) \in \sigma(\mathscr{Q})\right\}$ is a $\sigma$-algebra in $K$; we will show that it contains the generating sets for $\mathscr{B}(K)$. Given $f \in C(K)$, extend $f$ continuously to all of $X$. For each $\alpha \in R$,

$$
\begin{aligned}
\operatorname{st}_{Z}^{-1}(\{x \in K: f(x)<\alpha\}) & =\left\{z \in Z:{ }^{0}\left({ }^{*} f(z)\right)<\alpha\right\} \\
& =\bigcup_{\substack{n=1 \\
n \in N}}^{\infty}\left\{z \in Z:{ }^{*} f(x)<\alpha-\frac{1}{n}\right\} \in \sigma(\mathbb{Q}) .
\end{aligned}
$$

Thus, st $z$ is measurable. Given $f \in C(X)$ and $y \in Z,{ }^{0}\left({ }^{*} f(y)\right)=f(\operatorname{st}(y))$, so by Theorem 3 of [8], Equation 1 holds.

Corollary 1. Given $\nu \in{ }^{*} M_{f}(X)$, let $\nu_{X}=\left(\nu^{+}\right)_{X}-\left(\nu^{-}\right)_{X}$. Then $\nu_{X}$ is the standard part of $\nu$ with respect to the weak ${ }^{*}$ topology on $M(X)$. If $\nu={ }^{*} \mu$ for some $\mu \in M(X)$, then $\nu_{X}=\mu$.

In [3], Anderson and Rashid (using, in part, methods that originated in the proof of Theorem 4.6 of [9], described before Example 1), elaborated on their work in [1] and [13] to obtain a criterion for weak* convergence of a net of measures. Their proof of the measurability of the standard part map generalizes the proof in [9] and is essentially the proof of measurability used here. For a compact space $X$, their result can be directly obtained in a form that includes weak* cluster points by employing Theorem 1 and Robinson's characterization of cluster points and limit points of a net $\left\{U_{\alpha}\right\}_{\alpha \in D}$ (Theorems 4.2 .4 and 4.2 .5 of [15]). Recall that for a directed set $(D, \geqslant), \beta \in{ }^{*} D$ is infinite if $\beta \geqslant{ }^{*} \alpha$ for each standard $\alpha \in D$.

Corollary 2. Let $\left\{\nu_{\alpha}\right\}_{\alpha \in D}$ be a net in $M(X)$. For each infinite $\beta \in{ }^{*} D$ such that ${ }^{0}\left\|\nu_{\beta}\right\|<+\infty,\left(\nu_{\beta}\right)_{X}$ is a weak* cluster point of $\left\{\nu_{\alpha}\right\}$. These are the only weak* cluster points in $M(X)$ if $\nu_{\alpha}$ is a positive measure for each $\alpha \in D$ or if for some $r \in R$ and all $\alpha \in D,\left\|\nu_{\alpha}\right\| \leqslant r$. In the latter case, the weak* limit of $\left\{\nu_{\alpha}\right\}$ exists and is equal to $\nu_{1} \in M(X)$ if and only if, for each infinite $\beta \in{ }^{*} D, \nu_{1}=\left(\nu_{\beta}\right)_{X}$.

Given a nonnegative $\nu \in{ }^{*} M_{f}(X)$, set $\left.\mu(A)={ }^{0}\left(\nu{ }^{*} A\right)\right)$ for each $A \in \mathscr{B}(X)$. Then $\mu$ is $\sigma$-additive if and only if for some $\lambda \in M(X)$ and all $A \in \mathscr{B}(X)$,

$$
\mu(A)={ }^{0}\left(\nu\left({ }^{*} A\right)\right) \leqslant{ }^{*} \lambda\left({ }^{*} A\right)=\lambda(A) .
$$

For each $f \in C(X), \int_{X} f d \mu \simeq \int_{* X}^{*} f d \nu$. Therefore, $\mu$ is $\sigma$-additive if and only if $\mu=\nu_{X}$.

In actual practice, one may need to take the weak* standard part of an internal measure that has not come from the extension of a standard net. (See, for example, the use of Theorem 1 in [5].) On the other hand, using a standard net may simplify some arguments; consider the following variation of Anderson's [1] construction of Lebesgue measure.

EXAMPLE 2. It is well known that Lebesgue measure $\lambda$ represents the functional $f \rightarrow \int_{0}^{1} f(x) d x$ on $C([0,1])$. If we define a measure $\nu_{n}$ in terms of 
point masses $\delta_{x}$ at $x=1 / n, \ldots,(n-1) / n, 1$ by setting $\nu_{n}=(1 / n) \delta_{1 / n}$ $+\cdots+(1 / n) \delta_{1}$, then

$$
\lim _{n \rightarrow \infty} \int f d \nu_{n}=\int_{0}^{1} f(x) d x
$$

for each $f \in C[0,1]$. It follows that for any $\eta \in{ }^{*} N-N, \lambda$ is the weak* standard part of $\nu_{\eta}$, whence $\lambda=\left(\nu_{\eta}\right)_{[0,1]}$. For general Radon measures, the above construction can be obtained by using finite measurable partitions as in [7]; also see Anderson's construction in [2].

If $X$ is the product of compact spaces, $X=\prod_{\gamma \in I} X_{\gamma}$, then for $x=\left\{x_{\gamma}\right\}_{\gamma \in * I}$ $\epsilon^{*} X$,

$$
\operatorname{st}(x)=\left\{{ }^{0} x_{\gamma}\right\}_{\gamma \in I} .
$$

There is extensive application of Theorem 1 (established here) to product spaces in [5]. A similar application strengthens the results in [8] as follows.

Example 3. Let $X=\{0,1\}^{N}$. Given $\eta \in^{*} N-N$, let $Z$ consist of all internal hyperfinite sequences of 0 's and 1's of length $\eta$. By adjoining to each sequence in $Z$ an internally infinite sequence of 0 's, we may assume that $Z \subset{ }^{*} X$. In [8] $Z$ is used as a model for infinite coin tossing with each $z \in Z$, having internal $\nu$-probability $1 / 2^{\eta}$. If now one restricts the internal sequence $z(i)$ to the standard natural numbers, one obtains the standard part of $z$ with respect to the product topology on $X$. In this case, $\nu_{X}$ is the usual probability measure for coin tossing.

In general, let $X=\prod_{\gamma \in I} X_{\gamma}$ be a product of nonempty compact Hausdorff spaces with the product topology. If $H \subset I$, we write $X_{H}$ for $\Pi_{\gamma \in H} X_{\gamma}$; for $A \subset X_{H}$ and $B \subset X_{I-H}$ we write $A \times B$ for the set of elements of $X$ with projection on $X_{H}$ in $A$ and projection on $X_{I-H}$ in $B$. Let $\mu$ be a finitely additive probability measure on the algebra of Baire cyclinder sets $\mathscr{B}_{c}(X)$, i.e., the sets of the form $B \times X_{I-H}$ where $H$ is finite in $I$ and $B \in \mathscr{B}\left(X_{H}\right)$. Assume that for each finite set $H \subset I, \mu$ is countably additive on the $\sigma$-algebra $\mathscr{B}_{c}^{H}(X)=\left\{B \times X_{I-H}: B \in \mathscr{B}_{(}\left(X_{H}\right)\right\}$.

By the Kolmogorov extension theorem, $\mu$ has a countably additive extension $\tilde{\mu}$ on the collection of Baire sets $\mathscr{B}(X)$, which is the smallest $\sigma$-algebra in $X$ containing $\mathscr{B}_{c}(X)$. This can be proved by obtaining the extension of $\mu$ as follows: Fix a hyperfinite set $E \subset{ }^{*} I$ with ${ }^{*} \gamma \in E$ for each $\gamma \in I$. Fix an element $w \in X_{*_{I-E}}$, and let $Z=X_{E} \times\{w\}$. For each internal Baire set $A \subset X_{E}$, let $\nu(A \times\{w\})={ }^{*} \mu\left(A \times X_{*_{I-E}}\right)$. It is easy to see that $\nu_{X}$ is the desired extension of $\mu$. For the analogous result for the product of $\sigma$-compact, completely regular spaces, use Theorem 3 below.

Henson has shown in [4] that only Baire sets in $X$ have measurable inverse images with respect to the standard part map and a $\sigma$-algebra in ${ }^{*} X$ formed from an algebra of internal sets. Let $\mathscr{F}(X)$ denote the Borel sets in $X$. Each totally finite signed Baire measure $\mu$ on a compact set has a unique regular 
Borel extension which we shall denote by $\hat{\mu}$. Anderson has shown [2, Theorem 3.3] that for a standard $\mu \in M(X)$, each set $B$ in the $\hat{\mu}$-completion of $\mathscr{F}(X)$ has inverse image in the $\left({ }^{*} \hat{\mu}\right)_{0}$-completion of $\sigma\left({ }^{*} \mathscr{F}(X)\right)$ and $\hat{\mu}(B)=$ $\left({ }^{*} \hat{\mu}\right)_{0}\left(\mathrm{st}^{-1}(B)\right)$. We now show that for any positive internal $\nu \in{ }^{*} M_{f}(X)$ and any $B$ in $\mathscr{F}(X)$ or in the $\hat{\nu}_{X}$-completion of $\mathscr{F}(X)$, we have $\hat{\nu}_{X}(B)=\sup \left\{\nu_{0}(A)\right.$ : $\left.A \in{ }^{*} \mathscr{B}(X), \operatorname{st}(A) \subset B\right\}$. It will follow that $\mathrm{st}^{-1}(B)$ is measurable with respect to the $\nu_{0}$-completion of just $\sigma(* \mathscr{B}(X))$,

$$
\hat{\nu}_{X}(B)=\nu_{0}\left(\mathrm{st}^{-1}(B)\right)=\sup \left\{\nu_{0}(A): A \in * \mathscr{B}(X), A \subset \mathrm{st}^{-1}(B)\right\},
$$

and the family $\left\{\operatorname{st}^{-1}(B): B \in \mathcal{F}(X)\right\}$ is "universally" measurable in ${ }^{*} X$.

Theorem 2. Fix $Z \subset \subset^{*} X, \mathbb{Q}, \sigma(\mathbb{Q}), \nu, K=\operatorname{st}(Z)$, and $\Re(K)$ as in Theorem 1. Let $D$ be a compact subset of $K$. If $A$ is an internal subset of $Z$ with $\operatorname{st}(A) \subset D$ and $\nu(A)$ is defined (perhaps by extension of $\nu$ to an internal o-algebra containing $\mathcal{Q})$, then ${ }^{0}(\nu(A)) \leqslant \hat{\nu}_{K}(D)$. Moreover, there is an $A_{1} \in \mathbb{Q}$ with $\operatorname{st}\left(A_{1}\right)=D$ and $\nu_{0}\left(A_{1}\right)=\hat{\nu}_{K}(D)$. Thus if $B$ is any Borel set in $K$,

$$
\begin{aligned}
\hat{\nu}_{K}(B) & =\sup \left\{\hat{\nu}_{K}(D): D \text { compact, } D \subset B\right\} \\
& =\sup \left\{\nu_{0}(A): A \in \mathbb{Q}, \operatorname{st}(A) \subset B\right\} .
\end{aligned}
$$

Proof. Let $\alpha=\hat{\nu}_{K}(D)$. By the definition of $\hat{\nu}_{K}$, Theorem 1, and the Tietze Extension Theorem ([16, p. 148]),

$$
\alpha=\inf \left\{\int_{Z}{ }^{*} f d \nu: f \in C(X), 0 \leqslant f \leqslant 1, f \mid D \equiv 1\right\} .
$$

If $\nu(A)$ is defined and $\operatorname{st}(A) \subset D$, then for each standard $f \in C(X)$ with $0 \leqslant f \leqslant 1$ and $f \mid D \equiv 1$ we have ${ }^{*} f(a) \simeq 1$ for each $a \in A$. It follows that

$$
{ }^{0}(\nu(A))={ }^{0} \int_{A} d \nu={ }^{0} \int_{A}^{*} f d \nu \leqslant \int_{Z}^{*} f d \nu,
$$

whence ${ }^{0}(\nu(A)) \leqslant \alpha$.

On the other hand, we consider the internal relation $\Phi$, where the pair $(n, U)$ with $n \in{ }^{*} N$ and ${ }^{*} D \subset U \in{ }^{*} \mathcal{T}$ satisfies the $\Phi$ relation with respect to the function $f \in{ }^{*} C(X)$, i.e., $(n, U) \Phi f$, if and only if $0 \leqslant f \leqslant 1,\left.f\right|^{*} D \equiv$ $1, f \mid\left({ }^{*} X-U\right) \equiv 0$, and $\int_{z} f d \nu \geqslant \alpha-1 / n$. Since our enlargement is $\kappa$ saturated for $\kappa>\max \left(\aleph_{0}\right.$, card $\left.\mathcal{T}\right)$, and since the domain of the first argument of $\Phi$ contains and $\Phi$ is concurrent on the set of all standard pairs $\left(n,{ }^{*} U\right)$ with $n \in N$ and $D \subset U \in \mathcal{T}$, it follows from [11, p. 28] that there is an $f_{1} \in{ }^{*} C(X)$ such that $\left(n,{ }^{*} U\right) \Phi f_{1}$ for each standard pair $\left(n,{ }^{*} U\right)$ with $n \in N$ and $D \subset U \in \mathcal{T}$. Fix a positive infinitesimal $\varepsilon$, and let $A_{1}=\{z \in Z$ : $\left.f_{1}(z) \geqslant \varepsilon\right\}$. Then $\operatorname{st}\left(A_{1}\right)=D$ since $A_{1} \subset^{*} U$ for each standard open $U \supset D$ and $X$ is regular, and since $d \in A_{1}$ for each point $d \in D$. Moreover,

$$
\nu\left(A_{1}\right) \geqslant \int_{A_{1}} f_{1} d \nu \simeq \int_{Z} f_{1} d \nu \geqslant \alpha-\frac{1}{n}
$$

for each standard $n \in N$. Thus $\nu_{0}\left(A_{1}\right)=\alpha=\hat{\nu}_{K}(D)$. 
Corollary 3. If $\nu \in{ }^{*} M_{f}(X)$ is nonnegative and $B$ is in the $\hat{\nu}_{X}$-completion of $\mathcal{F}(X)$, then

$$
\hat{\nu}_{X}(B)=\sup \left\{\nu_{0}(A): A \in * \mathscr{B}(X), A \subset \operatorname{st}^{-1}(B)\right\} .
$$

Therefore, $\mathrm{st}^{-1}(B)$ is in the $\nu_{0}$-completion of $\sigma(* \mathscr{B}(X))$ and $\nu_{0}\left(\mathrm{st}^{-1}(B)\right)=\hat{\nu}_{X}(B)$. Moreover, the family $\left\{\mathbf{s t}^{-1}(B): B \in \mathcal{F}(X)\right\}$ is contained in every $\nu_{0}$-completion of $\sigma\left({ }^{*} \mathscr{B}(X)\right), \nu \in{ }^{*} M_{f}(X)$.

Proof. The corollary follows from the fact that $A \subset \mathrm{st}^{-1}(B)$ if and only if $\operatorname{st}(A) \subset B$ and $\hat{\nu}_{X}(B)+\hat{\nu}_{X}(X-B)=\hat{\nu}_{X}(X)=\nu_{0}\left({ }^{*} X\right)$.

Given $\nu \in{ }^{*} M_{f}(X)$, we have ${ }^{*} \nu_{X} \equiv{ }^{*}\left(\nu_{X}\right) \in{ }^{*} M_{f}(X)$. Since Borel sets have inverse images in the $\left({ }^{*} \nu_{X}\right)_{0}$-completion of $\sigma(* \mathscr{B}(X))$, it follows from either Corollary 3 here or Anderson's Theorem 3.3 of [2] that the same is true for a set $B$ in the $\hat{\nu}_{X}$-completion of $\mathscr{F}(X)$. Moreover,

$$
\left({ }^{*} \nu_{X}\right)_{0}\left(\mathrm{st}^{-1}(B)\right)=\hat{\nu}_{X}(B)=\nu_{0}\left(\mathrm{st}^{-1}(B)\right)
$$

Thus to measure the inverse image of Borel sets, it is sufficient to use standard measures and the completion of the $\sigma$-algebra formed from internal Baire sets.

For the rest of this note, we extend the above results to a noncompact space $X$. To do so, it is natural to consider the images of internal measures defined on not only the nonstandard extension of $X$ but the nonstandard extensions of compactifications of $X$. For example, if $X$ is the set rational numbers in $[-1,1]$ and $\nu$ is unit mass on an infinitesimal irrational number, then $\nu_{X}$ should be unit mass at 0 . We assume, therefore, that $\left(Y, \sigma_{Y}\right)$ is a compact Hausdorff space, that $X$ is a dense subspace of $Y$ (hence completely regular), and that our enlargement is $\kappa$-saturated where $\kappa>$ $\max \left(\boldsymbol{\kappa}_{0}\right.$, card $\left.\mathcal{T}_{Y}\right)$. Let $\mathscr{B}(Y)$ and $\mathscr{F}(Y)$ denote the collections of Baire sets in $Y$ and Borel sets in $Y$, respectively. Then

$$
\mathscr{B}(X)=\{A \cap X: A \in \mathscr{B}(Y)\} \quad \text { and } \mathscr{F}(X)=\{A \cap X: A \in \mathscr{F}(Y)\}
$$

are the collections of Baire and Borel sets in $X$. Let $M(Y)$ and $M(X)$ denote the signed Baire measures $\mu$ on $Y$ and $X$, respectively, with $\|\mu\|<+\infty$. Each $\mu \in M(Y)$ has a unique regular Borel extension $\hat{\mu}$; we call $\mu$ tight on $X$ if for any $\varepsilon>0$ in $R$, there is a compact $K_{\varepsilon} \subset X$ such that $|\hat{\mu}|\left(Y-K_{\varepsilon}\right)<\varepsilon$. When $\mu$ is tight on $X$, we extend $\hat{\mu}$ to $\mathscr{F}(X)$ by setting $\hat{\mu}(A \cap X)=\hat{\mu}(A)$ for each $A \in \mathscr{F}(Y)$. On the other hand, each $\rho \in M(X)$ determines a $\mu_{\rho} \in$ $M(Y)$ where $\mu_{\rho}(A)=\rho(A \cap X)$ for each $A \in \mathscr{B}(Y)$.

In [2], Anderson and Rashid showed that for an internal $\nu \in{ }^{*} M_{f}(X)$ (or even a finitely additive $\nu$ with $\left.{ }^{0}|\nu|\left({ }^{*} X\right)<+\infty\right)$ the $\sigma$-additive signed measure $\nu_{0}$ can be mapped via the standard part map onto a measure $\nu_{X} \in M(X)$ when ${ }^{*} X-\mathrm{st}^{-1}(X)$ is contained in a $\nu_{0}$-null set. Following Müller [12], they showed that this condition is equivalent to the tightness of the functional $f \rightarrow^{0} \int_{{ }^{*} X}{ }^{*} f d \nu$ defined on the space $C(X)$ of bounded continuous functions from $X$ into $R$. We shall extend these results to the case of a 
$\nu \in{ }^{*} M_{f}(Y)$ and show as well that each Borel set $B$ in $X$ has measurable inverse image with respect to the $\nu_{0}$-completion of $\sigma\left({ }^{*} \mathscr{B}(Y)\right)$ when $\nu_{0}\left({ }^{*} Y-\right.$ $\left.\mathrm{st}^{-1}(X)\right)=0$. Note that if $\nu$ is determined by an internal $\rho \in{ }^{*} M(X)$, i.e., $\nu(A)=\rho\left(A \cap{ }^{*} X\right)$ for each $A \in{ }^{*} \Re(Y)$, and ${ }^{*} Y-\mathrm{st}^{-1}(X)$ is contained in a $\nu_{0}$-null set (i.e., $\exists A \in *^{*}(Y)$ with $|\nu|(A) \simeq 0$ such that ${ }^{*} Y-$ st $^{-1}(X) \subset A$ ), then, ${ }^{*} X-\mathrm{st}^{-1}(X)$ is contained in a $\rho_{0}$-null set (i.e., $\exists A \in{ }^{*} \mathscr{B}(Y)$ with $|\rho|\left(A \cap \cap^{*} X\right) \simeq 0$ such that $\left.{ }^{*} X-\mathrm{st}^{-1}(X) \subset A \cap{ }^{*} X\right)$. We state the following result for Borel sets in $X$; the easy extension to completions of $\mathscr{F}(X)$ is left to the reader.

THEOREM 3. If $\nu \in{ }^{*} M_{f}(Y)$, then $\nu_{Y}$ is tight on $X$ if and only if ${ }^{*} Y-\mathrm{st}^{-1}(X)$ is contained in a $\nu_{0}$-null set. If $\nu_{Y}$ is tight on $X$ then for each $B \in \mathcal{F}(X), \mathrm{st}^{-1}(B)$ is in the $\nu_{0}$-completion of $\sigma\left({ }^{*} B(Y)\right)$ and $\hat{\nu}_{Y}(B)=\nu_{0}\left(\mathrm{st}^{-1}(B)\right)$. If $\nu$ is determined by $a \rho \in{ }^{*} M(X)$ and, after completion, $\rho_{0}\left({ }^{*} X-\mathrm{st}^{-1}(X)\right)=0$, then $\nu_{Y}$ is tight on $X$ and for each $B \in \mathscr{F}(X), \hat{\nu}_{Y}(B)=\rho_{0}\left(\operatorname{st}^{-1}(B) \cap{ }^{*} X\right)$.

Proof. Since $\nu=\nu^{+}-\nu^{-}$and $\nu_{Y}=\left(\nu^{+}\right)_{Y}-\left(\nu^{-}\right)_{Y}$, we may assume that $\nu=\nu^{+}$. If ${ }^{*} Y-\mathrm{st}^{-1}(X)$ is contained in a $\nu_{0}$-null set, then [8] for each $\varepsilon$ in $R^{+}$ there is an $A \in{ }^{*} \mathscr{B}(Y)$ such that $A \subset \mathrm{st}^{-1}(X)$ and $\nu_{0}\left({ }^{*} Y-A\right)<\varepsilon$. Let ${ }^{0} A=\operatorname{st}(A)$. By Theorem 2, $\hat{\nu}_{Y}\left({ }^{0} A\right) \geqslant \nu_{0}(A)$, so

$$
\hat{\nu}_{Y}\left(Y-{ }^{0} A\right)=\hat{\nu}_{Y}(Y)-\hat{\nu}_{Y}\left({ }^{0} A\right) \leqslant \nu_{0}\left({ }^{*} Y\right)-\nu_{0}(A)=\nu_{0}\left({ }^{*} Y-A\right)<\varepsilon .
$$

Since ${ }^{0} A$ is compact in $X$ and $\varepsilon$ is arbitrary in $R^{+}, \nu_{Y}$ is tight on $X$.

Assume now that $\nu_{Y}$ is tight on $X$. For any $\varepsilon>0$ in $R$ there is a compact set $K \subset X$ with $\hat{\nu}_{Y}(K)>\nu_{Y}(Y)-\varepsilon=\nu_{0}\left({ }^{*} Y\right)-\varepsilon$. By Theorem 2, there is an internal set $A \in * \mathscr{B}(Y)$ with $\operatorname{st}(A)=K$ such that $\nu_{0}(A)=\hat{\nu}_{Y}(K)>\nu_{0}\left({ }^{*} Y\right)-$ $\varepsilon$. Since $A \subset \mathrm{st}^{-1}(X)$ and $\varepsilon$ is arbitrary in $R^{+},{ }^{*} Y-\mathrm{st}^{-1}(X)$ is contained in a $\nu_{0}$-null set.

If $\nu_{Y}$ is tight on $X$, then for each $A \in \mathscr{F}(Y)$,

$$
\mathrm{st}^{-1}(A \cap X)=\mathrm{st}^{-1}(A) \cap \mathrm{st}^{-1}(X)
$$

which is measurable with respect to the $\nu_{0}$-completion of $\sigma\left({ }^{*} \mathscr{B}(Y)\right)$. Also, by definition $\hat{\nu}_{Y}(A \cap X)=\hat{\nu}_{Y}(A)$, and by Corollary 3 ,

$$
\hat{\nu}_{Y}(A)=\nu_{0}\left(\mathrm{st}^{-1}(A)\right)=\nu_{0}\left(\mathrm{st}^{-1}(A) \cap \mathrm{st}^{-1}(X)\right)=\nu_{0}\left(\mathrm{st}^{-1}(A \cap X)\right) .
$$

If $\nu$ is determined by a $\rho \in{ }^{*} M(X)$ and, after completion, $\rho_{0}\left({ }^{*} X-\mathrm{st}^{-1}(X)\right)$ $=0$, then after choosing $\varepsilon>0$ in $R$ and extending $\nu$ and thus $\nu_{0}$, one can find an internal $A \subset{ }^{*} X \cap \mathrm{st}^{-1}(X)$ with $\nu_{0}\left({ }^{*} Y-A\right)<\varepsilon$. Let ${ }^{0} A=\operatorname{st}(A)$. Again by Theorem $2, \hat{\nu}_{Y}\left({ }^{0} A\right) \geqslant \nu_{0}(A)$ so

$$
\hat{\nu}_{Y}\left(Y-{ }^{0} A\right) \leqslant \nu_{0}\left({ }^{*} Y-A\right)<\varepsilon .
$$

Thus $\nu_{Y}$ is tight on $X$. Moreover, if $A \in \mathscr{F}(Y)$, then

$$
\hat{\nu}_{Y}(A \cap X)=\nu_{0}\left(\mathrm{st}^{-1}(A \cap X)\right)=\rho_{0}\left(\left(\mathrm{st}^{-1}(A \cap X)\right) \cap{ }^{*} X\right) .
$$

Assume, finally, that $Y$ is the Stone-Čech compactification of $X$ and $\phi$ is an internal functional on ${ }^{*} C(X)$ with ${ }^{0}\|\phi\|<+\infty$. Then $\phi$ is represented by 
some $\nu \in{ }^{*} M_{f}(Y)$, and $\nu_{Y}$ represents the standard part of $\phi$ in the weak topology. We may replace $\nu_{Y}$ with a tight measure on $X$ if ${ }^{*} Y-\operatorname{st}^{-1}(X)$ is contained in a $\nu_{0}$-null set.

\section{REFERENCES}

1. R. M. Anderson, A nonstandard representation for Brownian motion and Itô integration, Israel J. Math 25 (1976), 15-46.

2. Star-finite representations of measure spaces (to appear).

3. R. M. Anderson and Salim Rashid, A nonstandard characterization of weak convergence, Proc. Amer. Math. Soc. 69 (1978), 327-332.

4. C. W. Henson, Analytic sets, Baire sets, and the standard part map, J. London Math. Soc. (to appear).

5. L. L. Helms and P. A. Loeb, Applications of nonstandard analysis to spin models, J. Math. Anal. App. (to appear).

6. G. Herglotz, Über Potenzreihen mit positivem, reellem Teil im Einheitskreis, Ber. Verhandl. Sächs Akad. Wiss. Leipzig Math.-Phys. Klasse 63 (1911), 501-511.

7. P. A. Loeb, A nonstandard representation of measurable spaces, $L_{\infty}$, and $L^{*}$, Contributions to Nonstandard Analysis (W. A. J. Luxemburg and A. Robinson, Eds.), North-Holland, Amsterdam, 1972, pp. 65-80.

8. Conversion from nonstandard to standard measure spaces and applications in probability theory, Trans. Amer. Math. Soc. 211(1975), 113-122.

9. Applications of nonstandard analysis to ideal boundaries in potential theory, Israel J. Math. 25 (1976), 154-187.

10. A generalization of the Riesz-Herglotz theorem on representing measures, Proc. Amer. Math. Soc. 71 (1978), 65-68.

11. W. A. J. Luxemburg, A general theory of monads, Applications of Model Theory to Algebra, Analysis, and Probability (W. A. J. Luxemburg, Ed.), Holt, Rinehart, and Winston, New York, 1969, pp. 18-86.

12. D. W. Müller, Nonstandard proofs of invariance principles in probability theory, Applications of Model Theory to Algebra, Analysis, and Probability (W. A. J. Luxemburg, Ed.), Holt, Rinehart, and Winston, New York, 1969, pp. 186-194.

13. S. Rashid, Economies with infinitely many traders, Ph.D. Dissertation, Yale University, May 1976.

14. F. Riesz, Sur certaines systèmes singuliers d'équations intégrales, Ann. Sci. École Norm. Sup. 28 (1911), 33-62.

15. A. Robinson, Non-standard analysis, North-Holland, Amsterdam, 1966.

16. H. L. Royden, Real analysis, Macmillan, New York, 1968.

17. K. D. Stroyan and W. A. J. Luxemburg, Introduction to the theory of infinitesimals, Series on Pure and Applied Mathematics, vol. 72, Academic Press, New York, 1976.

Department of Mathematics, University of Illinois, Urbana, Illinois 61801 